\title{
Design and Analysis of a Multi-Legged Robot with Pitch Adjustive Units
}

\author{
Qiang Ruan ${ }^{1}$, Jianxu Wu ${ }^{2^{*}}$ and Yan-an Yao ${ }^{1 *}$
}

\begin{abstract}
The paper proposes a novel multi-legged robot with pitch adjustive units aiming at obstacle surmounting. With only 6 degrees of freedom, the robot with 16 mechanical legs walks steadily and surmounts the obstacles on the complex terrain. The leg unit with adjustive pitch provides a large workspace and empowers the legs to climb up obstacles in large sizes, which enhances the obstacle surmounting capability. The pitch adjustment in leg unit requires as few independent adjusting actuators as possible. Based on the kinematic analysis of the mechanical leg, the biped and quadruped leg units with adjustive pitch are analyzed and compared. The configuration of the robot is designed to obtain a compact structure and pragmatic performance. The uncertainty of the obstacle size and position in the surmounting process is taken into consideration and the parameters of the adjustments and the feasible strategies for obstacle surmounting are presented. Then the 3D virtual model and the robot prototype are built and the multi-body dynamic simulations and prototype experiments are carried out. The results from the simulations and the experiments show that the robot possesses good obstacle surmounting capabilities.
\end{abstract}

Keywords: Mechanical leg, Multi-legged robot, Obstacle surmounting, Adjustive pitch, Leg unit

\section{Introduction}

The demands for the mobile robots are growing with the spreading scope of human activity. However, on the complex uneven terrains, the mobility of these traditional platforms is restricted. Leg robots contact the ground in separated areas, and the leg robots are relatively less affected by the bad road [1]. The supporting feet on the ground provide frictions as the traction forces. The swinging legs lift up and stride forwards at the same time. The supporting and swinging legs exchange periodically to perform the walking movements.

Since the mid-twentieth century, with the increasing research of the legged robots, numerous leg prototypes have been developed. To achieve practical application, there are many important matters to be considered, such

\footnotetext{
*Correspondence: 13116343@bjtu.edu.cn; yayao@bjtu.edu.cn

${ }^{1}$ Key Laboratory of Vehicle Advanced Manufacturing, Measuring

and Control Technology, Ministry of Education, Beijing Jiaotong

University, Beijing 100044, China

${ }^{2}$ Department of Mechanical Engineering, Tsinghua University, Beijing 100084, China
}

as the gait planning [2, 3], the balance walking [4], the load carrying capacity [5] and the power consumption $[6,7]$. Many popular leg robots were designed and constructed in a bionic way [8]. They usually have four limbs and the mechanical legs have obvious hip, knee and ankle joints, just like leg animals. Every leg of the robot has two DoFs or more, so the leg is very flexible and the workspace of the foot end is large, thus the robot maintains its balance and overcomes the complex environments. This kind of the bio-inspired leg robots adopts serial mechanisms as the legs. Bigdog $[9,10]$ and AlphaDog $[11]$ by Boston Dynamics are quadruped robots powered by gasoline engine. Bigdog had 16 active joints driven by hydraulic actuators, and it climbed $35^{\circ}$ slope with loose scree-like surface. Bigdog was used in US army to carry goods. ANYmal $[12,13]$ by ETH Zurich is an electricpowered quadruped robot with series elastic actuators (SEAs). ANYmal climbed $20^{\circ}$ slope, $45^{\circ}$ stairs, $350 \mathrm{~mm}$ step, $200 \mathrm{~mm}$ obstacle and surmounted $250 \mathrm{~mm}$ gap. It was developed to support humans in dirty, dangerous and dull work. MIT Cheetah 2 [14] realized high speed 


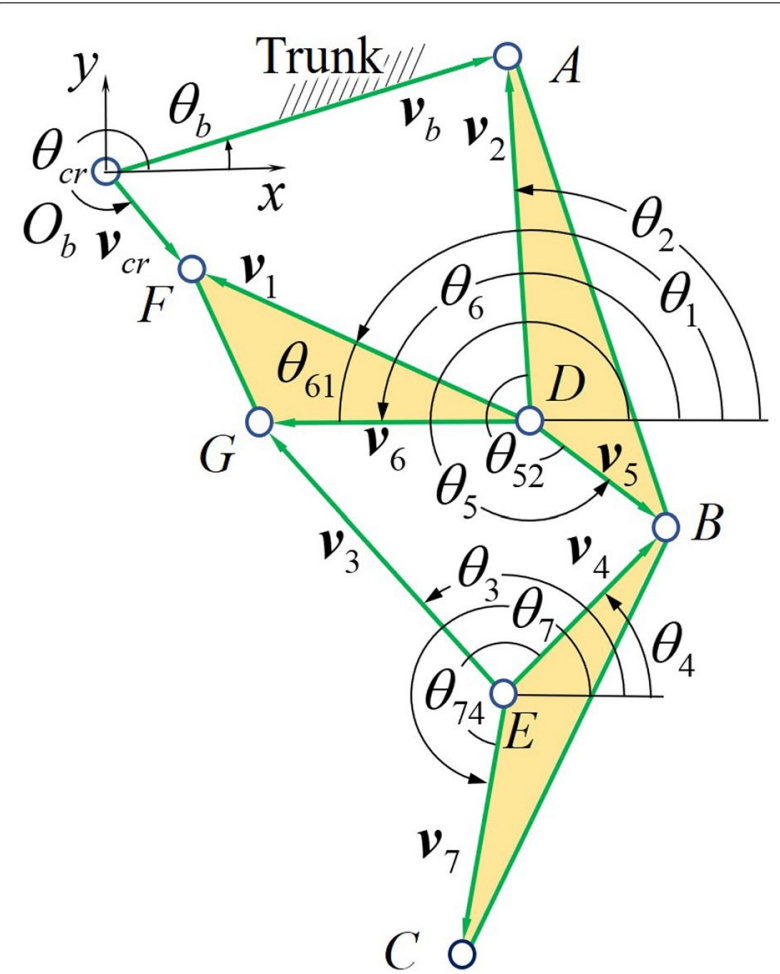

Figure 1 Schematic diagram of the leg mechanism

running and autonomous jumping over the obstacles 400 $\mathrm{mm}$ in height, which was $80 \%$ of the leg length. There are also some robots using parallel mechanisms as mechanical legs. Octopus robot [15] used parallel mechanisms as the mechanical legs and have the heavy load carrying capacity. Octopus robot climbed the $150 \mathrm{~mm}$ step. WL-16R [16] is a load-carrying biped robot with parallel mechanical legs which could dynamically walk. WL$16 \mathrm{R}$ had $340 \mathrm{~mm}$ step height to overcome the obstacles. These robots usually have large numbers of actuators to be controlled precisely and simultaneously. The calculated motion of the robot relies on the feedback from the sensors, such as IMU, force sensors and laser radar. As a result, the control algorithm is complex and the hardware is expensive.

Designed with a different conception, some walking robots have close-loop mechanical legs. With the reasonable configuration and size of the link mechanism, the mechanical leg generates the trajectory that fits for walking. Two or more legs share the common trunk link and crank link to form leg group and they are driven by one actuator to generate the walking motion [17]. Robots with close-loop mechanical legs or leg groups usually have good load carrying capabilities with high energy efficiency [18]. The legged robot with fewer DoFs benefits much from the simplicity of the control system. Hoecken
Table 1 Target points

\begin{tabular}{llll}
\hline $\boldsymbol{i}$ & $\boldsymbol{P}_{\boldsymbol{i}}$ & & $\boldsymbol{\theta}_{\mathrm{cri}}(\mathrm{rad})$ \\
\cline { 2 - 4 } & $\boldsymbol{x}_{\boldsymbol{i}}(\mathbf{m m})$ & $\boldsymbol{y}_{\boldsymbol{i}}(\mathbf{m m})$ & \\
\hline 1 & 310 & -470 & $1.78 \pi$ \\
2 & 220 & -470 & $1.56 \pi$ \\
3 & 130 & -470 & $1.34 \pi$ \\
4 & 40 & -470 & $1.12 \pi$ \\
5 & 310 & -350 & $0.22 \pi$ \\
\hline
\end{tabular}

mechanism [19], Theo Jansen mechanism [20], Chebyshev mechanism [21] and Klann mechanism [22] are able to walk with only one actuator. These multi-legged robots have good load-carrying capacity [23] and high-speed running potentiality $[24,25]$. They are low in cost without expensive controllers and the actuators. For the leg group in the robot, only one continuously rotating motor drives the legs to stride towards, and the control system can be quite simple. However, robot of this style has the disadvantage in the adaptivity in the rough environments for the changeless foot end point trajectory.

Adjustments to the changeless trajectory enhanced the adaptivity. In our previous work, the reconfigurable legs [26] and the legs with variable topologies [27] both generated the adjustable foot trajectories in different modes. The partial adjustments in the legs greatly increased the amount of DoFs, and the small-scaled adjustments couldn't adapt to the obstacles in large sizes.

To surmount the large-sized obstacles, the large-scaled adjustability is one of the necessary conditions. Mobile robot with articulated body has the larger workspaces than the ordinary ones. The articulated robots with wheels or tracks performed well on the complex ground. MULE [28] by Lockheed Martin had six actuated arms between the wheels and the body and it can overcome large obstacles. MULE crossed $1000 \mathrm{~mm}$ gap, $500 \mathrm{~mm}$ obstacle, and climbed $22^{\circ}$ traverse side slopes. Packbot [29] is a tracked robot with articulated body that has all-terrain adaptability. Packbot climbed the $43^{\circ}$ ascend or descend slope, $40^{\circ}$ lateral slope and $254 \mathrm{~mm}$ stairs. Research on the multi-legged robot with articulated body has been less explored yet.

The remaining parts of the paper are organized as follows. In Section 2, different kinds of the leg units with adjustive pitch is proposed and analyzed based on the kinematic analysis of a single mechanical leg. The foot end point movements of the different leg units are obtained and compared. Then the robot layout is given. The obstacle surmounting strategy is presented in Section 3. To test the effectiveness and the performance of the design, model simulations and prototype experiments 
Table 2 Link geometry parameters

\begin{tabular}{llllll}
\hline Length & Value $(\mathbf{m m})$ & Length & Value $(\mathbf{m m})$ & Angle & Value (rad) \\
\hline$v_{\mathrm{b}}$ & 270.42 & $v_{4}$ & 187.17 & $\theta_{\mathrm{b}}$ & 0.6545 \\
$v_{\mathrm{cr}}$ & 78.55 & $v_{5}$ & 107.83 & $\theta_{52}$ & 3.6633 \\
$v_{1}$ & 236.74 & $v_{6}$ & 215.74 & $\theta_{61}$ & 1.5521 \\
$v_{2}$ & 169.19 & $v_{7}$ & 217.13 & $\theta_{74}$ & 4.1087 \\
$v_{3}$ & 193.25 & & & & \\
\hline
\end{tabular}

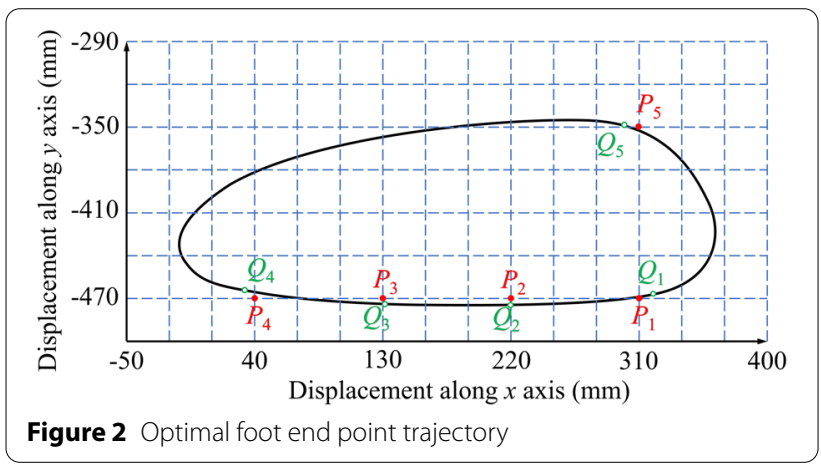

are carried out in Section 4. Based on the data from the simulations and experiments, Section 5 gives the conclusion that the novel robot possesses the large-sized obstacle surmounting capability the meets the expectation.

\section{Leg Unit}

The walking motion needs the cooperative movements of the legs, and a single leg is not able to accomplish walking. To gain the surmounting capability over the obstacles in large sizes, the walking unit mainly consists of the mechanical legs and the actuated adjusting joints. There are different combination forms depending on the amount of the legs and the positions of the regulating joints which show different performances.

\subsection{Mechanical Leg}

All the legs of the robot are in the same configuration. The mechanical leg is a Watt Chain mechanism, which is a planar link mechanism with a single degree of freedom. To simplify the calculation, the trunk link $O_{b} A$ is temporarily fixed. The rectangular coordinate system is setup with the origin $O_{b}$ at the rotating center of the crank $O_{b} F$. The schematic diagram of the leg mechanism is shown in Figure 1 and the geometry parameters of the links are marked. In the schematic diagram, $\theta_{52}, \theta_{61}, \theta_{74}, \theta_{b}$ and $v_{i}(i=b, \mathrm{cr}, 1,2, \ldots, 7)$ are the geometry parameters of the links. The points $O_{b}, A, B, D, E, F$ and $G$ are revolute joints. $C$ is the foot end point. $\theta_{i}(i=1,2, \ldots, 7)$ are the orientation angles of the corresponding vectors, and they are the unknown variables to be solved. $\theta_{\mathrm{cr}}$ is the orientation angle of the crank and it is the independent variable.

According to the topological configuration of the mechanical leg, the vector loop equations are obtained as:

$$
\left\{\begin{array}{l}
\boldsymbol{v}_{\mathrm{cr}}-\boldsymbol{v}_{1}+\boldsymbol{v}_{2}-\boldsymbol{v}_{b}=0 \\
-\boldsymbol{v}_{3}+\boldsymbol{v}_{4}-\boldsymbol{v}_{5}+\boldsymbol{v}_{6}=0
\end{array}\right.
$$

The vector loop equations are expanded and then solved with trigonometric transform and elimination method. All the expressions of the unknown variables are obtained by solving the vector loop equations. The solutions are the long and complicated expressions. To express the solutions concisely, some intermediate variables, such as $p_{i j}(i=1,2,3,4 ; j=1,2,3)$ and $q_{i j}(i=1,2$, $3 ; j=1,2)$ are used. The solutions are expressed as:

$$
\begin{aligned}
& \theta_{i}=2 \tan ^{-1}\left(\frac{p_{i 2}+\sqrt{p_{i 1}^{2}+p_{i 2}^{2}-p_{i 3}^{2}}}{p_{i 1}-p_{i 3}}\right),(i=1,3), \\
& \theta_{i}=2 \tan ^{-1}\left(\frac{p_{i 2}-\sqrt{p_{i 1}^{2}+p_{i 2}^{2}-p_{i 3}^{2}}}{p_{i 1}-p_{i 3}}\right),(i=2,4), \\
& \theta_{i}=\theta_{j}+\theta_{i j},((i, j)=(5,2),(6,1),(7,4)) .
\end{aligned}
$$

The related intermediate variables are expressed as:

$$
\begin{aligned}
& \left(\begin{array}{ll}
q_{11} & q_{12} \\
q_{21} & q_{22} \\
q_{31} & q_{32}
\end{array}\right)=\left(\begin{array}{ccc}
\boldsymbol{v}_{\mathrm{cr}} c \theta_{\mathrm{cr}}-\boldsymbol{v}_{b} c \theta_{b} & \boldsymbol{v}_{6} c \theta_{6}-\boldsymbol{v}_{5} c \theta_{5} \\
\boldsymbol{v}_{\mathrm{cr}} s \theta_{\mathrm{cr}}-\boldsymbol{v}_{b} s \theta_{b} & \boldsymbol{v}_{6} s \theta_{6}-\boldsymbol{v}_{5} s \theta_{5} \\
\boldsymbol{v}_{1}^{2}-\boldsymbol{v}_{2}^{2} & \boldsymbol{v}_{3}^{2}-\boldsymbol{v}_{4}^{2}
\end{array}\right), \\
& \left(\begin{array}{lll}
p_{11} & p_{12} & p_{13} \\
p_{21} & p_{22} & p_{23} \\
p_{31} & p_{32} & p_{33} \\
p_{41} & p_{42} & p_{43}
\end{array}\right)=\left(\begin{array}{ccc}
2 \boldsymbol{v}_{2} q_{11} & 2 \boldsymbol{v}_{2} q_{21} & q_{11}^{2}+q_{21}^{2}-q_{31} \\
-2 \boldsymbol{v}_{11} q_{11} & -2 \boldsymbol{v}_{1} q_{21} & q_{11}^{2}+q_{21}^{2}+q_{31} \\
2 \boldsymbol{v}_{4} q_{12} & 2 \boldsymbol{v}_{4} q_{22} & q_{12}^{2}+q_{22}^{2}-q_{32} \\
-2 \boldsymbol{v}_{3} q_{12} & -2 \boldsymbol{v}_{3} q_{22} & q_{12}^{2}+q_{22}^{2}+q_{32}
\end{array}\right) .
\end{aligned}
$$

In Eq. (5), $s$ and $c$ are the abbreviations of sin and $\cos$ functions correspondingly. The movement of the foot end point $C$ is important, and it decides the capability to a large extent. According to the topological configuration, it is obtained that:

$$
\overrightarrow{O_{b} C}=v_{\mathrm{cr}}-\boldsymbol{v}_{1}-\boldsymbol{v}_{4}+\boldsymbol{v}_{5}+\boldsymbol{v}_{7} .
$$

The equation is expanded and the coordinate of the foot end point $C\left(x_{C}, y_{C}\right)$ in the reference frame $x O_{b} y$ is expressed as:

$$
\left\{\begin{array}{l}
x_{C}=\boldsymbol{v}_{\mathrm{cr}} c \theta_{\mathrm{cr}}-\boldsymbol{v}_{1} c \theta_{1}-\boldsymbol{v}_{4} c \theta_{4}+\boldsymbol{v}_{5} c \theta_{5}+\boldsymbol{v}_{7} c \theta_{7}, \\
y_{C}=\boldsymbol{v}_{\mathrm{cr}} s \theta_{\mathrm{cr}}-\boldsymbol{v}_{1} s \theta_{1}-\boldsymbol{v}_{4} s \theta_{4}+\boldsymbol{v}_{5} s \theta_{5}+\boldsymbol{v}_{7} s \theta_{7} .
\end{array}\right.
$$




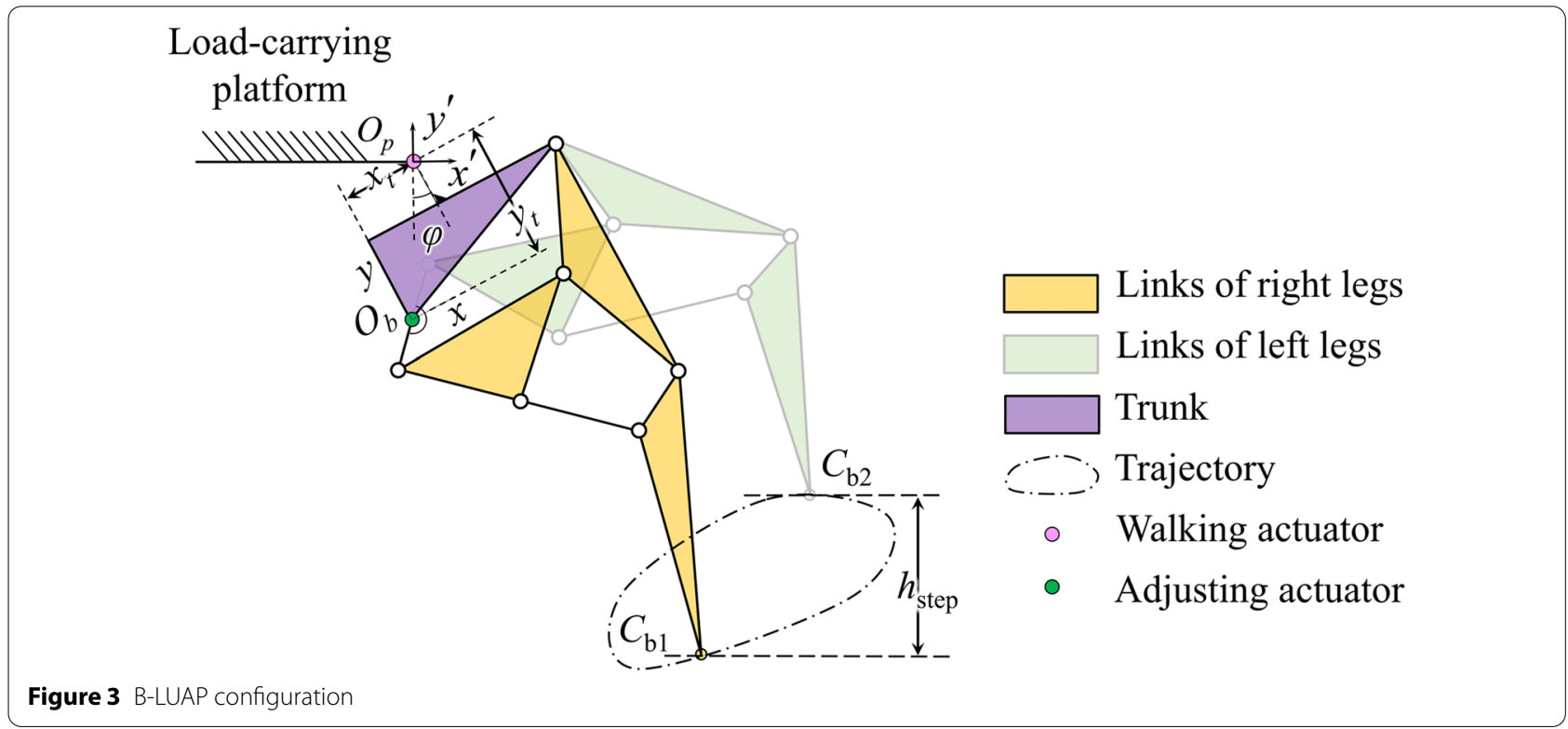

To gain the better mechanical properties of the leg, the geometry parameters of the links and the foot end point trajectory is to be optimized. Five target points $P_{i}(i=1$, $2,3,4,5)$ are given in Table $1 . P_{i}(i=1,2,3,4)$ have the same altitudes, horizontal distance intervals and crank phase intervals, so that the trajectory in the lower section can be smooth. Point $P_{5}$ is set to control the height of the trajectory.

$Q_{i}(i=1,2,3,4,5)$ are the five points in the corresponding crank phases on the trajectory. They are expected to be as close to the target points as possible. The objective function is expressed as:

$$
f=\operatorname{minimize}\left(\sum_{i=1}^{5}\left|P_{i} Q_{i}\right|^{2}\right) .
$$

As the points $Q_{i}(i=1,2,3,4,5)$ are on the foot end point trajectory, they are subject to:

$$
\left\{\begin{array}{l}
x_{Q_{i}}=x_{c}\left(\theta_{\text {cri }}\right) \\
y_{Q_{i}}=y_{c}\left(\theta_{\text {cri }}\right)
\end{array},(i=1,2,3,4,5) .\right.
$$

The geometry parameters of the links are obtained by solving the optimization problem. The optimal value of the object function is $f=291.63 \mathrm{~mm}^{2}$. The corresponding link geometry parameters are listed in Table 2 .

With the optimal parameters of the links, the foot end point trajectory is plotted in Figure 2.

\subsection{Leg Unit with Adjustive Pitch}

Each mechanical leg has only one degree of freedom, and it has the fixed shape of the foot end trajectory. As a result, the step height, step length and all other

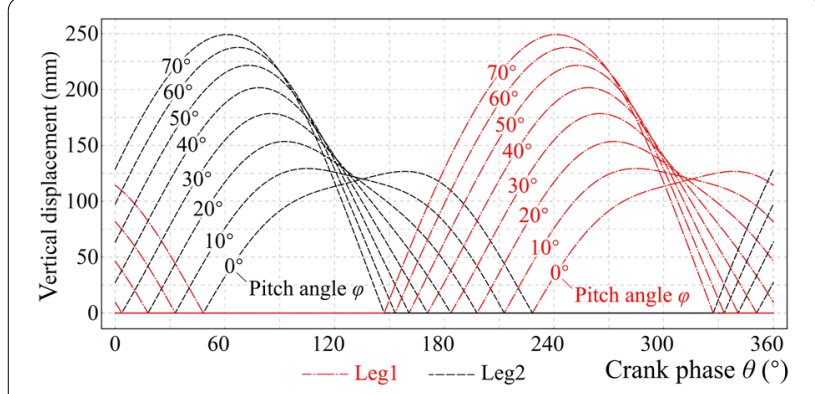

Figure 4 Foot end vertical displacements of B-LUAP

parameters of the mechanical legs are constant values. However, the leg robot needs the surmounting capabilities over the obstacles in different sizes. To achieve various parameters, adjustments are essential.

To make the foot end trajectories changeable, one possible way is to replace the rigid links with the actuated joints to adjust the sizes, which is in the single leg level. The adjustments are achieved by introducing at least one actuator to each mechanical leg. The advantage is that different legs can be regulated independently. Yet the disadvantages are listed as follows. Firstly, the adjustment in the single leg level is in a small scale. It can hardly improve the performance significantly for the geometric size limits. Secondly, the amount of the regulating actuators is large in the multi-legged robot, which may lead to the significant increasement of the robot mass and the complexity. Then, as a single leg is not able to accomplish walking motions, the legs need to cooperate with each other, and the adjustments have to be synchronically controlled by the expensive servo motors, in case of 


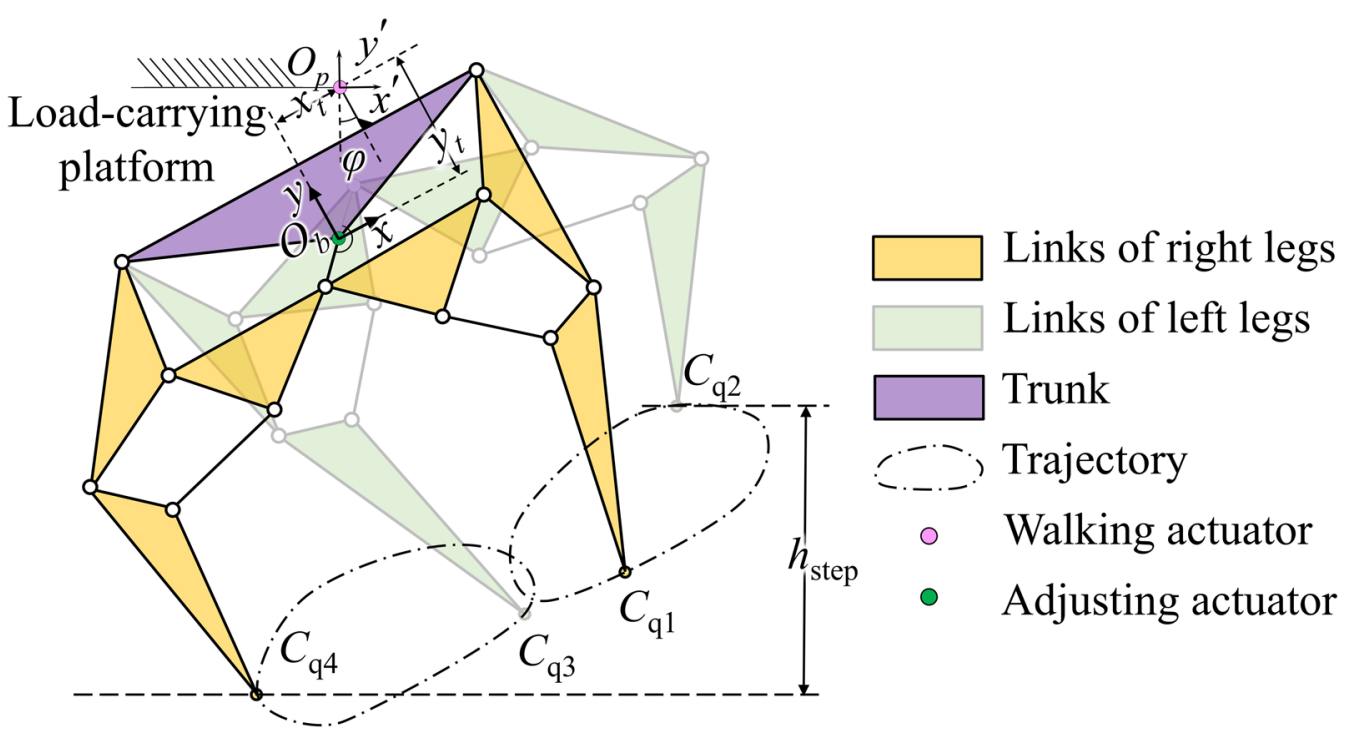

Figure 5 Q-LUAP configuration

disturbing the walking gaits. Lastly, the actuators have to be installed in the narrow spaces, and they have to swing or rotate with the adjacent links, as a result, the lectotype of the actuators is limited, and the wiring is difficult.

Another way is to adjust the attitude of the leg group as a whole. The leg group is the integration of two or more legs with a common trunk. The regulating object is the common trunk instead of the links in the leg. The benefit of regulating in the leg group level is that, the adjustment position is equivalent to the hip joint or above, and the amplification effects of the adjustments are more obvious. As the legs are in groups, the amount of the regulating actuators decreases a lot comparing with the one that regulates in the single leg level. The grouped legs share the same regulating actuator.

The attitude of the leg group is expressed with three angles. The adjustments of yaw and roll angles don't affect much on the obstacle surmounting capability of the robot. The pitch angle adjustment makes differences. The appropriate pitch angle lifts some of the foot end points up, and the corresponding legs gain enough altitude to the ground to surmount the obstacles. The leg group and the pitch adjustment together make up of the leg unit with adjustive pitch (LUAP).

With the different amount of the legs in a leg unit, there are different configurations for the LUAPs. Taking the compactness of the mechanism and the effectiveness of the walking motion into consideration, we propose the biped LUAP and the quadruped LUAP, which are to be analyzed.

\subsubsection{Biped Leg Unit with Adjustive Pitch}

The biped leg unit with adjustive pitch (B-LUAP) has a leg group with 2 abreast legs in the parallel planes. The 2 legs share the common trunk and the crank shaft. The cranks have the $\pi$ phase difference so that the movement is symmetric.

The right leg is marked Leg 1 and the left one is marked Leg 2. The corresponding foot end points are marked $C_{\mathrm{b} 1}$ and $C_{\mathrm{b} 2}$, as shown in Figure 3.

In the reference frame $x O_{b} y, O_{p}\left(x_{t}, y_{t}\right)$ is a revolute joint connecting the platform and the trunk with the actuator. $x^{\prime} \mathrm{O}_{p} y^{\prime}$ is a reference frame fixed on the platform.

Kinematic analysis of the B-LUAP is based on a single leg. It is appointed that the positive walking direction in the paper towards right and the corresponding positive crank rotating direction is clockwise. The phase of B-LUAP is set to be $\theta=2 \pi-\theta_{\text {cr }}$, where $\theta_{\text {cr }}$ is the crank phase of Leg 1 . The legs are projected to the vertical plane, and we get coordinates of the foot end points $C_{\mathrm{b} 1}$ and $C_{\mathrm{b} 2}$. They are expressed as:

$$
\left(\begin{array}{c}
C_{\mathrm{b} 1} \\
C_{\mathrm{b} 2}
\end{array}\right)=\left(\begin{array}{cc}
x_{C}(-\theta)-x_{t} & y_{C}(-\theta)-y_{t} \\
x_{C}(\pi-\theta)-x_{t} & y_{C}(\pi-\theta)-y_{t}
\end{array}\right) R(\varphi) .
$$

In the equation, $R(\phi)$ is the rotation matrix. As the motions of the legs have the symmetry about $y$-axis, $O_{p}$ is set to be along $y$-axis $\left(x_{t}=0\right)$ to reduce the peak torque on $O_{p}$. The value of $y_{t}$ also affects the peak torque on $O_{p}$ in the obstacle surmounting process. The smaller value of $y_{t}$ leads to the shorter arm of force and the lower torque payload, which improves the performance. Considering 


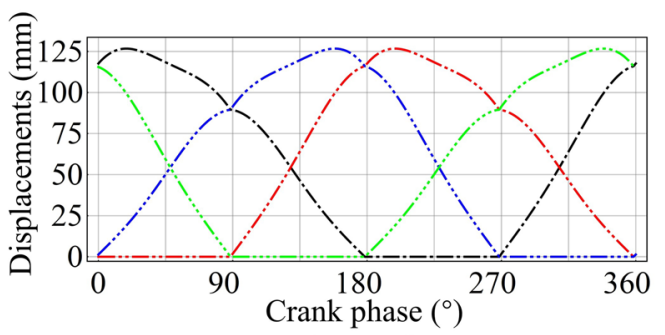

(a) $\varphi=0^{\circ}$

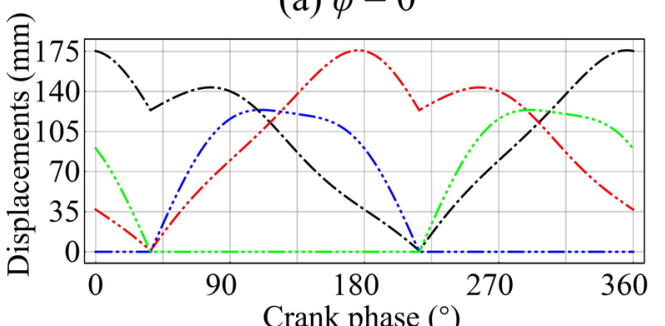

(c) $\varphi=7.4^{\circ}$

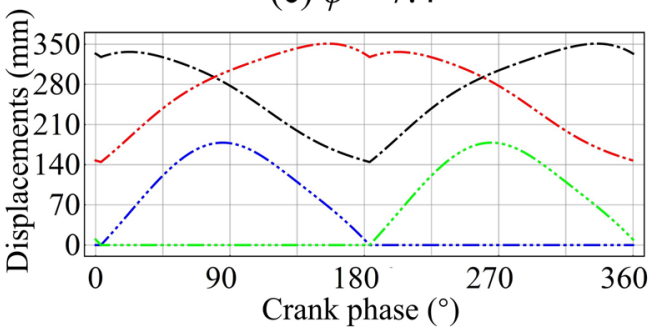

(e) $\varphi=30^{\circ}$

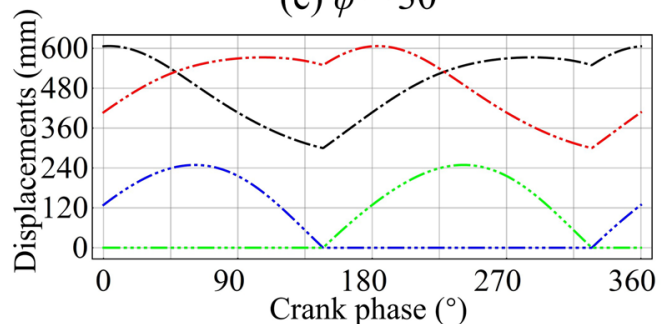

(g) $\varphi=70^{\circ}$

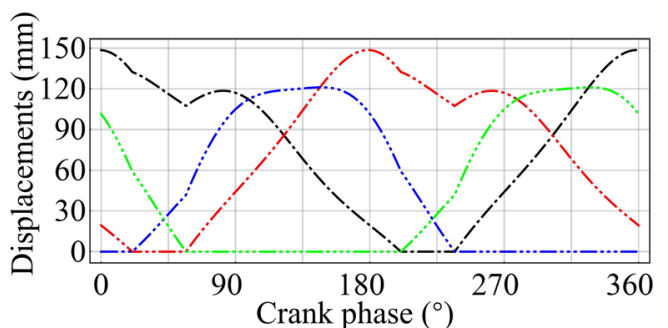

(b) $\varphi=4^{\circ}$

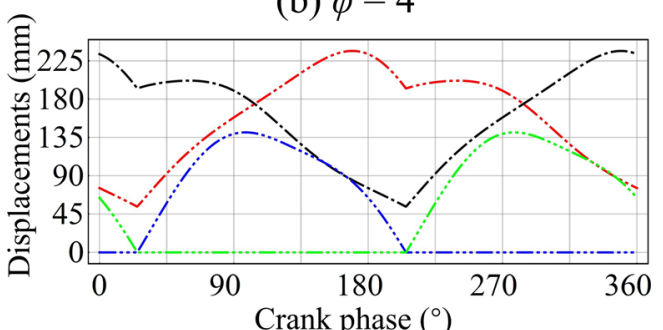

(d) $\varphi=15^{\circ}$

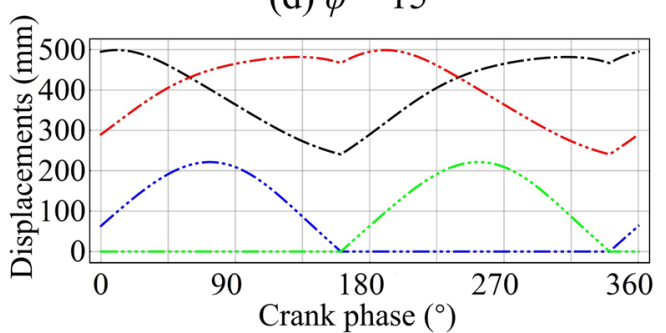

(f) $\varphi=50^{\circ}$

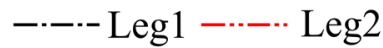

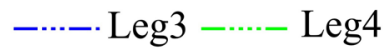

Figure 6 Foot end vertical displacements of Q-LUAP

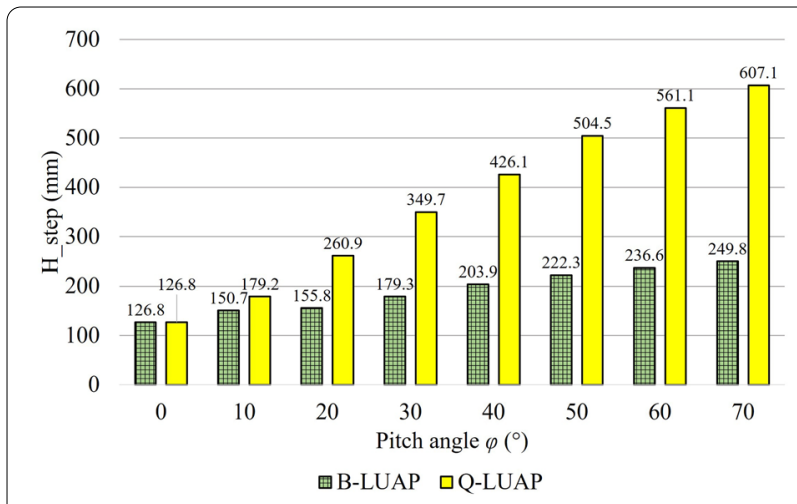

Figure 7 Step height comparison

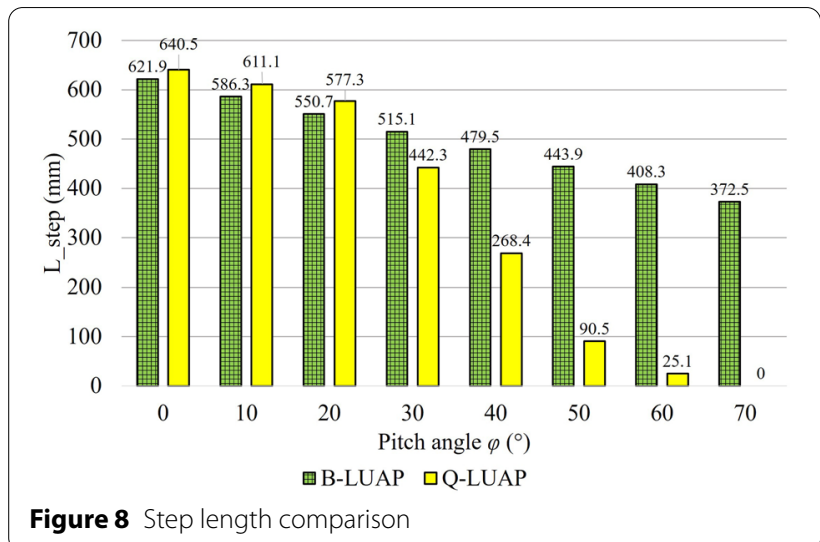




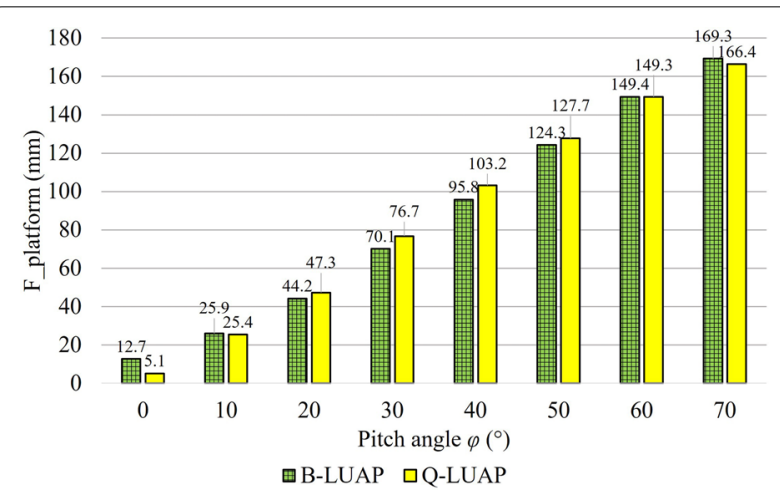

Figure 9 Platform height fluctuation comparison

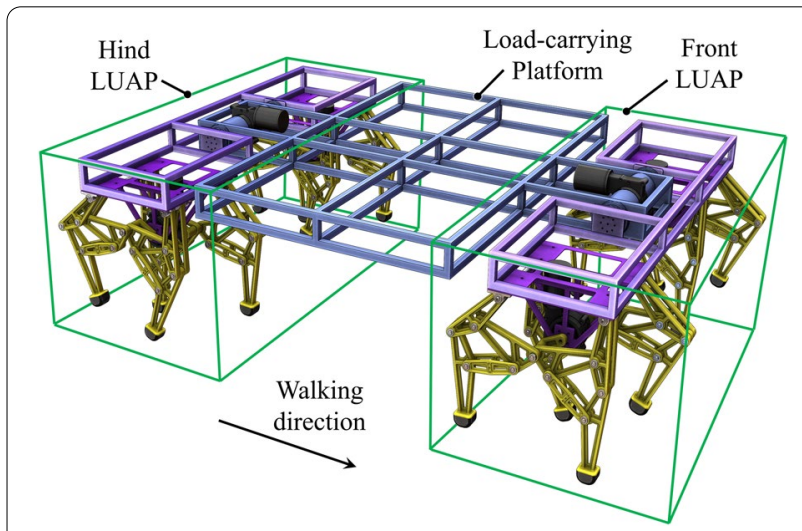

Figure 10 Leg unit layout

the size limits and the performance synthetically, $y_{t}=$ $-30 \mathrm{~mm}$ is decided.

The vertical displacements between the foot end points and the ground have the direct effects on the obstacle surmounting capability. It is supposed that the trunk keeps level and remains a constant attitude when walking on the flat ground. The foot end points of the supporting legs have no relative slippages to the ground. The leg-ground contact points are supposed to be the foot end points regardless of the geometry of the foot. $L G V D_{i}$ is the leg-ground vertical displacement of Leg $i$. For BLUAP, $L G V D_{i}$ is expressed as:

$$
L G V D_{i}=y_{C_{\mathrm{b} i}}-\min \left(y_{C_{\mathrm{b} 1}}, y_{C_{\mathrm{b} 2}}\right),(i=1,2) .
$$

The leg-ground vertical displacements vary with the phase $\theta$ and the pitch angle $\varphi$. The data for B-LUAP in one period of the phase $\theta$ is plotted and shown in Figure 4.

The legs touch the ground in turn. The proportion of the supporting phase occupies exactly half of one period, regardless of the pitch angle $\varphi$. With the increase of the pitch angle $\varphi$, the maximum leg-ground vertical displacement is increasing, and the obstacle surmounting capability is better. When $\varphi=0^{\circ}$, the leg is in the normal walking mode and it is able to reach $126.8 \mathrm{~mm}$ in height, and when $\varphi=70^{\circ}$, it is able to reach $249.8 \mathrm{~mm}$.

\subsubsection{Quadruped Leg Unit with Adjustive Pitch}

The quadruped leg unit with adjustive pitch (Q-LUAP) integrates four mechanical legs, and the hind ones are towards the opposite direction to the front ones sharing a common crank. The crank on the left has a $\pi$ phase difference from the right one in order to achieve coordinated walking. The four legs are marked Leg 1 to Leg 4 from the front right one. The foot end points of the four legs are counter-clockwise marked $C_{\mathrm{q} i}(i=1,2,3,4)$ correspondingly, as shown in Figure 5.

For Q-LUAP, the kinematic analysis is similar to the one for the B-LUAP configuration. It is also appointed that the positive walking direction is towards right and the positive crank rotating direction is clockwise. The phase of Q-LUAP has the same appointment as B-LUAP. The coordinates of the foot end points $C_{\mathrm{q} i}(i=1,2,3$ and 4) are obtained, and they are expressed as:

$$
\left(\begin{array}{c}
C_{\mathrm{q} 1} \\
C_{\mathrm{q} 2} \\
C_{\mathrm{q} 3} \\
C_{\mathrm{q} 4}
\end{array}\right)=\left(\begin{array}{cc}
x_{C}(-\theta)-x_{t} & y_{C}(-\theta)-y_{t} \\
x_{C}(\pi-\theta)-x_{t} & y_{C}(\pi-\theta)-y_{t} \\
-x_{C}(\theta)-x_{t} & y_{C}(\theta)-y_{t} \\
-x_{C}(\pi+\theta)-x_{t} & y_{C}(\pi+\theta)-y_{t}
\end{array}\right) \boldsymbol{R}(\varphi) .
$$

In the equation, $\boldsymbol{R}(\varphi)$ is also the rotation matrix. The leg-ground vertical displacement $L G V D_{i}$ for is expressed as:

$$
L G V D_{i}=y_{C_{\mathrm{q} i}}-\min _{j=1,2,3,4}\left(y_{C_{\mathrm{q} j}}\right),(i=1,2,3,4) .
$$

The leg-ground vertical displacements of Q-LUAP in one period of the phase $\theta$ is plotted and shown in Figure 6.

The maximum leg-ground vertical displacement grows with the pitch angle $\varphi$, but it has the faster growth. $\varphi$ $=7.4^{\circ}$ is the critical value. When $\varphi<7.4^{\circ}$, all the four mechanical legs have supporting phases. When $\varphi>7.4^{\circ}$, the two front legs (Leg 1 and Leg 2) are lifted up without supporting phases. Theoretically when $\varphi=40^{\circ}$ the legs climb up to $426.1 \mathrm{~mm}$ and when $\varphi=70^{\circ}$, the legs climb up to $607.1 \mathrm{~mm}$.

The trajectory is a closed oval curve with a relatively flat bottom. The points $Q_{i}(i=1,2,3,4,5)$ on the trajectory are close to the target points $P_{i}(i=1,2,3,4,5)$ correspondingly. 


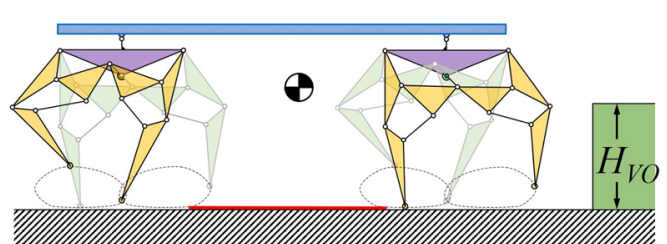

(a) State 0

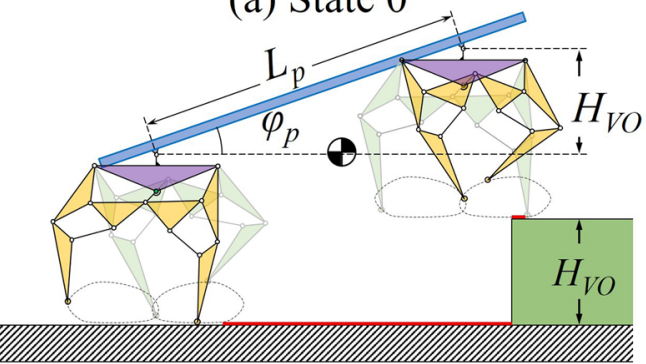

(c) State 2

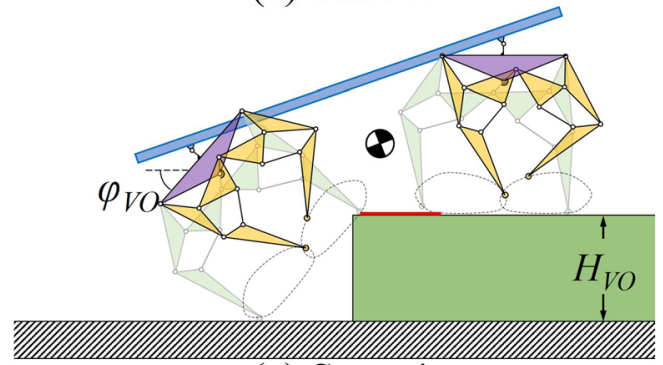

(e) State 4

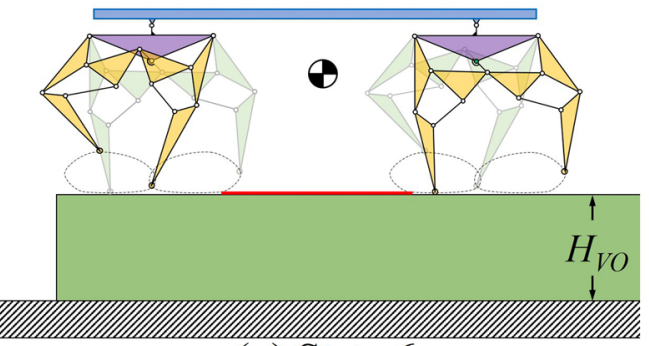

(g) State 6

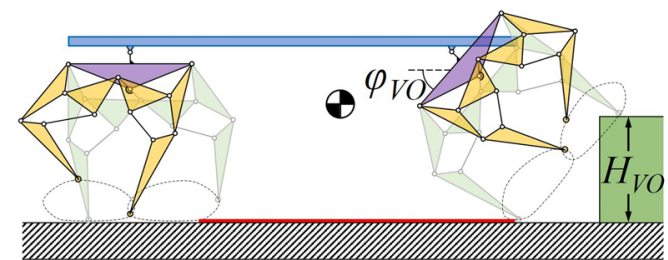

(b) State 1

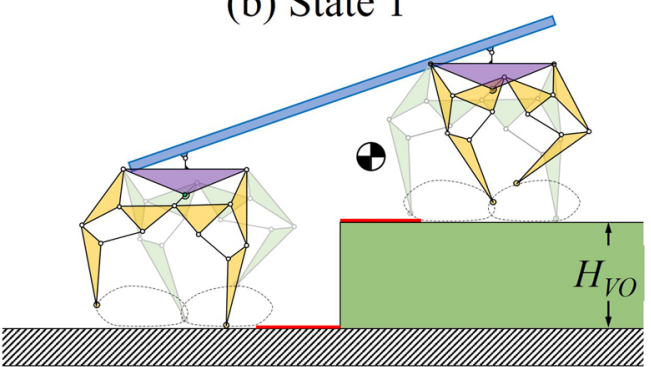

(d) State 3

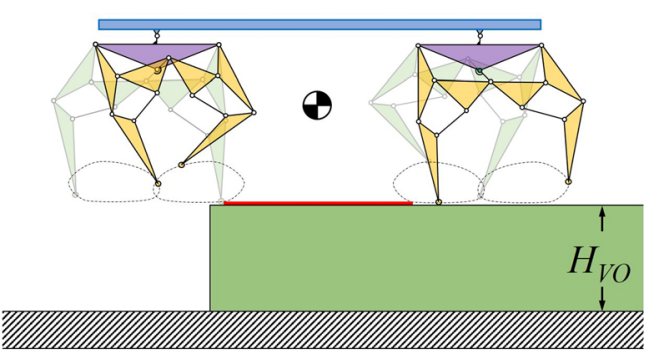

(f) State 5

Load-carrying platform

Links of right legs

Links of left legs

Trunk

Obstacle

Ground

Minimal supporting area

Robot's center of mass

Figure 11 Vertical obstacle surmounting strategy

Table 3 Vertical obstacle surmounting parameters

\begin{tabular}{llll}
\hline Stages & Platform pitch $\varphi_{\boldsymbol{p}}$ & $\begin{array}{l}\text { Hind LUAP } \\
\text { trunk pitch } \varphi_{\boldsymbol{H}}\end{array}$ & $\begin{array}{l}\text { Front LUAP } \\
\text { trunk pitch } \varphi_{\boldsymbol{F}}\end{array}$ \\
\hline State 0 & 0 & 0 & 0 \\
State 1 & 0 & 0 & $\varphi_{V O}$ \\
State 2, 3 & $\sin ^{-1}\left(H_{V O} / L_{P}\right)$ & 0 & 0 \\
State 4 & $\sin ^{-1}\left(H_{V O} / L_{P}\right)$ & $\varphi_{V O}$ & 0 \\
State 5, 6 & 0 & 0 & 0 \\
\hline
\end{tabular}

\subsection{Parameter Comparison}

The parameter comparisons between the B-LUAP and the Q-LUAP configurations are in aspects of the step height (H_step), the step length (L_step) and the height fluctuation range of the load-carrying platform (F_platform) at different pitch angles $\varphi$. The results are shown in Figures 7, 8 and 9 correspondingly.

The step height is the altitude distance between the highest foot end point and the lowest one in a crank cycle. The value of step height reflects the ultimate obstacle surmounting capability. When the pitch angle increases, the step heights for both B-LUAP and Q-LUAP grow, and the growth is much faster for Q-LUAP, which means that 
Table 4 Vertical obstacle surmounting scenarios

\begin{tabular}{lll}
\hline Scenarios & Characteristics & Passing capacity \\
\hline S1 & $H_{V O}<h_{\min }\left(\varphi_{V O}\right)$ & Passable \\
S2 & $H_{V O}>h_{\max }\left(\varphi_{V O}\right)$ & Impassable \\
S3 & $H_{V O} \in\left[h_{\min }\left(\varphi_{V O}\right), h_{\max }\left(\varphi_{V O}\right)\right]$ & $\begin{array}{l}\text { Passable with potential } \\
\text { impact }\end{array}$ \\
\hline
\end{tabular}

Q-LUAP can surmount the larger obstacles. In aspect of the step height, Q-LUAP is superior to B-LUAP.

The step length is the horizontal displacement of the load-carrying platform in one crank cycle. This parameter indicates the walking velocity. When the pitch angle increases, the step lengths both decrease, and the decreasement for Q-LUAP is more obvious. Slowing down before obstacle surmounting is the regular operation, and the larger obstacle requires the slower movement to ensure the stablity. In aspect of the step length, Q-LUAP is better.

The height fluctuation range of the load-carrying platform is the max interval length of vertical displacement of the platform, which indicates the jolt of the robot. When the pitch angle increases, the values for both
B-LUAP and Q-LUAP also increase without obvious differences. When the robot walks on the flat ground, $\varphi$ $=0^{\circ}$. The vertical jolt is only $5.1 \mathrm{~mm}$ for Q-LUAP and it is $12.7 \mathrm{~mm}$ for B-LUAP. The Q-LUAP walks more smoothly on the flat ground than the other.

As a conclusion for the comparison, the Q-LUAP has the advantages over the B-LUAP, both for surmounting obstacles and smoothly walking on the flat ground. The Q-LUAP is selected to build the robot.

\subsection{Leg Unit Layout}

The structure layout of the robot adjusts to the functions. The robot is designed to do the delivery jobs on the ground with obstacles. There are two major functions. One is that the robot has the relatively strong capability in obstacle surmounting, and the other is that the robot has the smooth gait, the load carrying capability and enough space to restore the goods.

For the balance and walking stability, four Q-LUAPs are arranged to the corners of the robot. The four Q-LUAPs together support the load carrying platform. As the overall size of the robot is not large, the left Q-LUAPs and the right ones overcome the similar obstacles. The abreast

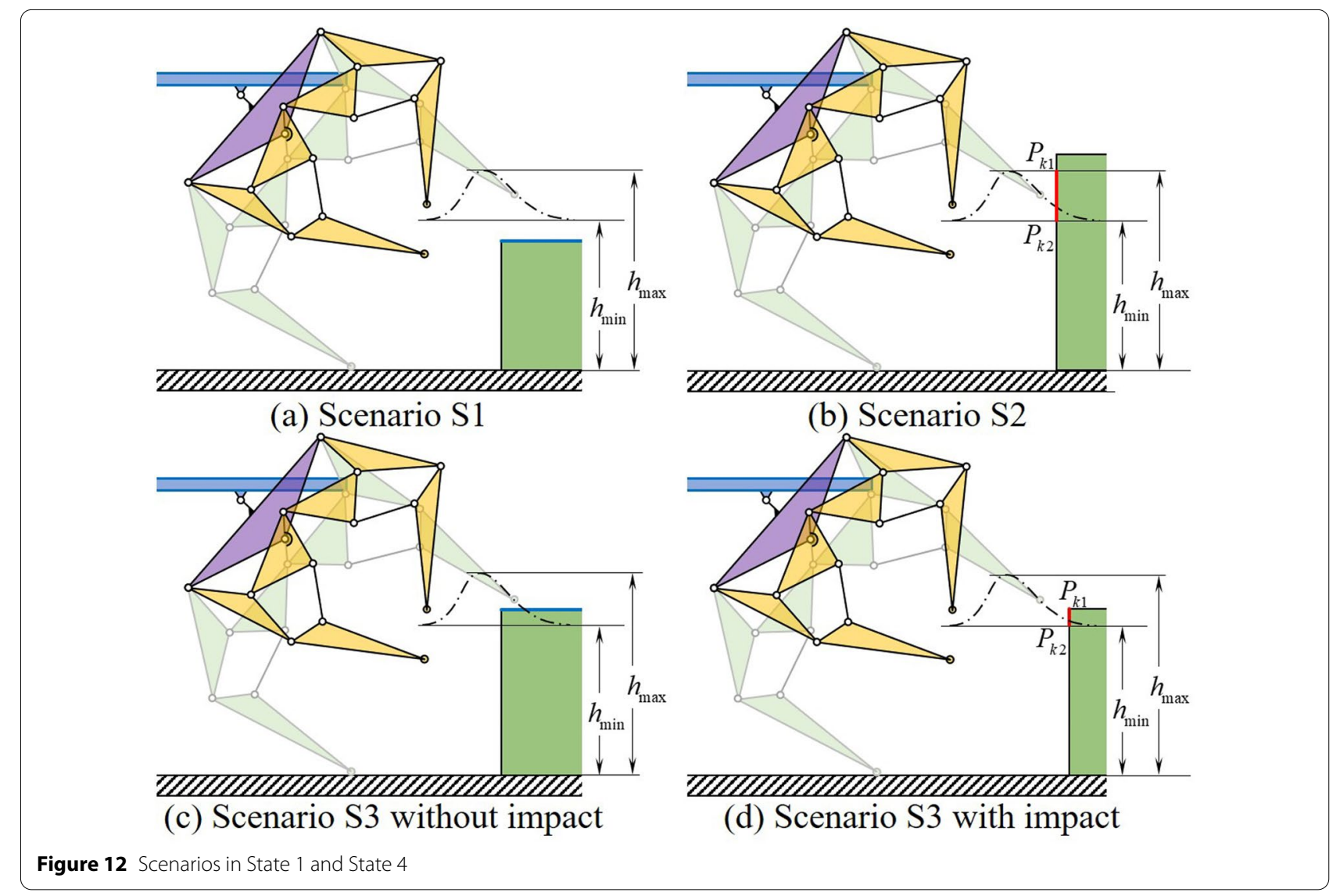




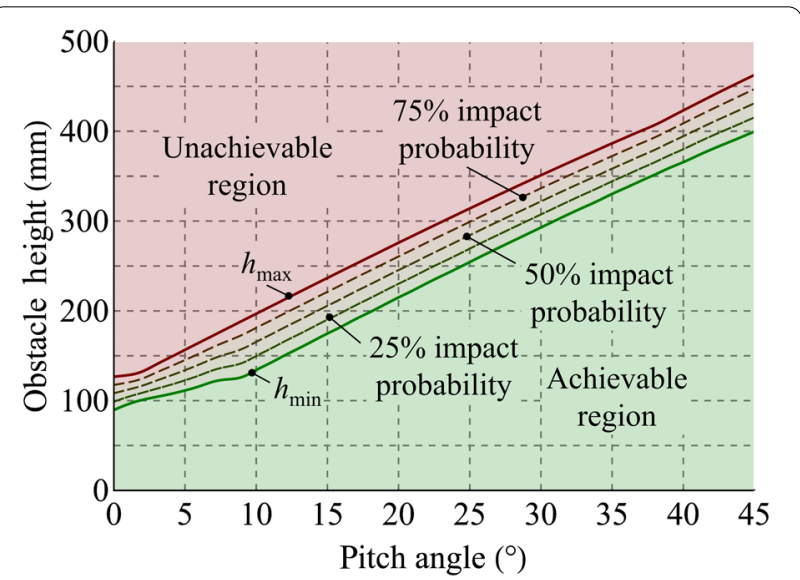

Figure 13 Pitch angle-obstacle height curves

Q-LUAPs share the same trunk and the same pitch adjusting actuator, and they form two LUAPs in the front and at the hind of the robot, as shown in Figure 10.

When the phases of the quadruped units are the same, the projections are coincident and only four legs are visible. In this case, the analysis of LUAP is the same as the Q-LUAP.

\section{Vertical Obstacle Surmounting Strategy}

The vertical obstacle is the step which is a cuboid shaped obstacle fixed on the ground. For the symmetry of the robot, walking forward and backward have the same performances. Climbing up and down are the reverse processes with similar situations, so only the strategy for climbing up is given.

The whole process is divided into six sub processes by seven states, the seven states are shown in Figure 9. The pitch of the trunk, the phase of the crank and the pitch of
Table 5 Simulation parameters

\begin{tabular}{llllll}
\hline Model & \multicolumn{2}{l}{ Robot parameters } & & \multicolumn{2}{l}{ Other settings } \\
\cline { 2 - 3 } & Pitch angle $\left(^{\circ}\right)$ & Mass $(\mathbf{k g})$ & & Gravity $\left(\mathbf{m} / \mathbf{s}^{2}\right)$ & $\begin{array}{l}\text { Precision } \\
(\mathbf{s t e p s} / \mathbf{s})\end{array}$ \\
\hline Model 0 & 0 & 180 & & -9.8 & 400 \\
Model 1 & $-40 \sim 40$ & & & \\
\hline
\end{tabular}

the platform are given in each state. All the pitch angles are relative to the ground.

State 0 is the initial state, as shown in Figure 11a. From State 0 to State 1 , the hind LUAP keeps still. The pitch angle of the trunk in the front LUAP increases, and at the same time, the front LUAP steps forward in order to reduce the slippage. In the sub process, the hind legs of the front LUAP support on the ground, and lift the front legs until the front legs step on the top of the vertical obstacle, as shown in Figure 11b. From State 1 to State 2 , the pitch angle of the front LUAP decreases until the trunk is horizontal, and the trunk of the hind one keeps horizontal. The hind LUAP steps forward in order to reduce the slippage, as shown in Figure 11c. From State 2 to State 3, the pitch angles keep unchanged and both LUAPs step forward, as shown in Figure 11d. From State 3 to State 4, the front LUAP keeps still, the hind LUAP steps forward and the pitch angle of the trunk increases until the front legs step on the top of the vertical obstacle, as shown in Figure 11e. From State 4 to State 5, the front trunk keeps horizontal and the hind one decreases the pitch angle until it is horizontal. At the same time, the front LUAP steps forward to reduce the slippage, as shown in Figure 11f. From State 5 to State 6, both the LUAPs step forward until the robot is entirely on the top, as shown in Figure 11g.

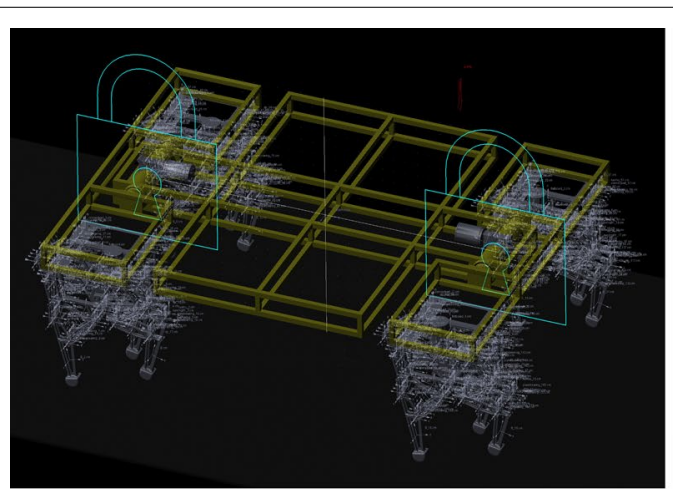

(a) Model 0 with rigid frame

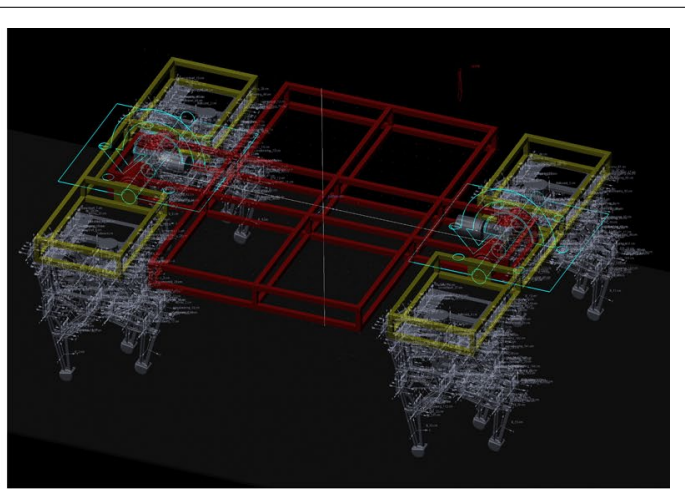

(b) Model 1 with LUAPs

Figure 14 Simulation models 


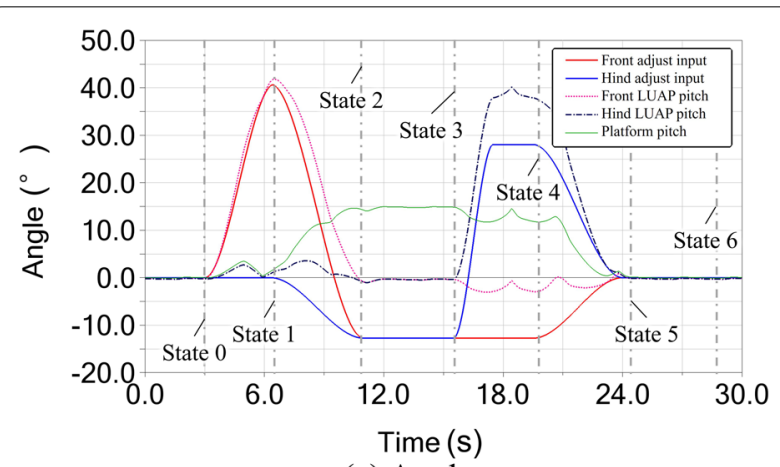

(a) Angles

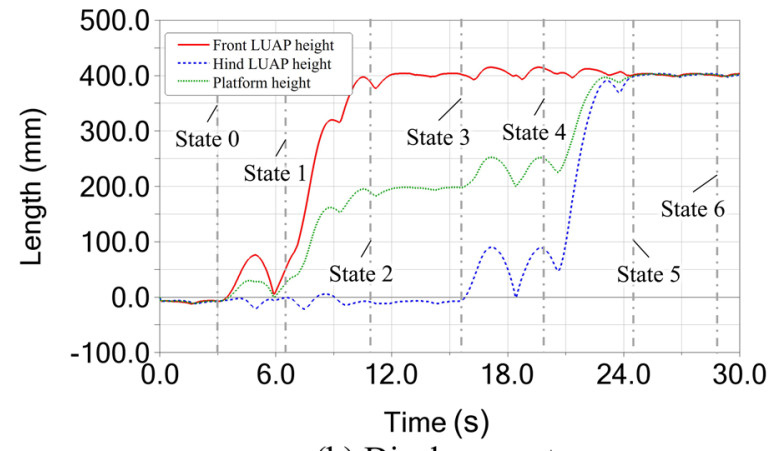

(b) Displacements

Figure 15 Simulation results of Model 1

The sixteen-legged robot is a self-stabilizing mechanism. The robot uses kinematic gaits to climb the obstacles, and the center of mass locates above the minimal supporting area in each state, so the robot keeps stable during the whole obstacle-climbing process.

According to the vertical obstacle surmounting strategy, parameters in the different states are listed in Table 3. Notably, the pitch angles in the table are in the world reference frame.

Theoretically, the necessary condition to achieve obstacle surmounting is that in State 1 and State 4, the height of the vertical obstacle is not higher than the max step height of the LUAP, for both the front and the hind ones, and that is:

$$
h_{\text {step }}\left(\varphi_{V O}, \theta_{V O}\right) \geq H_{V O} .
$$

$\theta_{V O}$ is the crank phase in obstacle surmounting State 1 of the front LUAP. The obstacle usually has the random distance from the robot, so $\theta_{V O}$ is a random variable which is subject to uniform distribution from 0 to $2 \pi$. There are three possible scenarios S1, S2 and S3, as listed in Table 4.

In the table, $h_{\min }$ and $h_{\max }$ are the lower and upper boundaries of the step height range at a certain pitch angle. They are expressed as:

$$
\begin{aligned}
& h_{\max }\left(\varphi_{V O}\right)=\max _{0 \leq \theta_{V O}<2 \pi}\left(h_{\text {step }}\left(\varphi_{V O}, \theta_{V O}\right)\right), \\
& h_{\min }\left(\varphi_{V O}\right)=\min _{0 \leq \theta_{V O}<2 \pi}\left(h_{\text {step }}\left(\varphi_{V O}, \theta_{V O}\right)\right) .
\end{aligned}
$$

In Scenario S1, the vertical obstacle is lower than the bottom of the reaching foot and the robot is able to step on the top of the obstacle and then surmount it, as shown in Figure 12a. In Scenario S2, all the feet are under the top of the obstacle, and the reaching foot impacts on the vertical surface, so the robot has no chance to surmount the obstacle as shown in Figure 12b. In Scenario S3, the performance depends on the crank phase in State 1 and State 4. Some ranges of the crank phases make the reaching foot step on the top of the obstacle, and the robot can pass the obstacle without impacts as shown in Figure 12c. Other ranges make the reaching foot impact on the vertical surface. After the impact, the crank continues to rotate, and the supporting foot slips backwards until the step height reach the top of the obstacle, then the reaching leg steps on the obstacle and the robot passes the obstacle as shown in Figure 12d.

Based on the scenarios, the relationship between the pitch angle and the obstacle height is obtained and shown in Figure 13.

To surmount the vertical obstacles in different heights, the pitch angle adjustment must be compatible. Insufficient pitch angle leads to the impact or even fail to surmount the obstacle. On the other hand, the excessive pitch angle lead to the unnecessary energy loss, the extra structure stress and the poor efficiency. The suggested pitch angle should be subject to the green solid line in Figure 11. For further illustration, the pitch angle $\varphi_{V O}$ stay $0^{\circ}$ when the obstacle height is lower than $89.2 \mathrm{~mm}$. When the obstacle is $250 \mathrm{~mm}$ in height, the pitch angle $\varphi_{V O}$ should be adjusted to $25^{\circ}$.

The range of the pitch adjustment $\varphi_{V O}$ is also limited by many factors such as the mechanical interferences, torques and the structure strength.

\section{Simulations and Experiments}

\subsection{Simulation}

The simulations were carried out in ADAMS software. A virtual model (Model 1) and a controlled virtual model (Model 0) were established as shown in Figure 14. The only difference of the two models is the pitch angle adjustability.

For surmounting vertical obstacle and walking on the slopes, the simulations were carried out in pairs. Simulations of Model 0 are the reference trials.

The inputs of the pitch adjustments are smoothly interpolated between the adjacent states, to avoid the sudden 


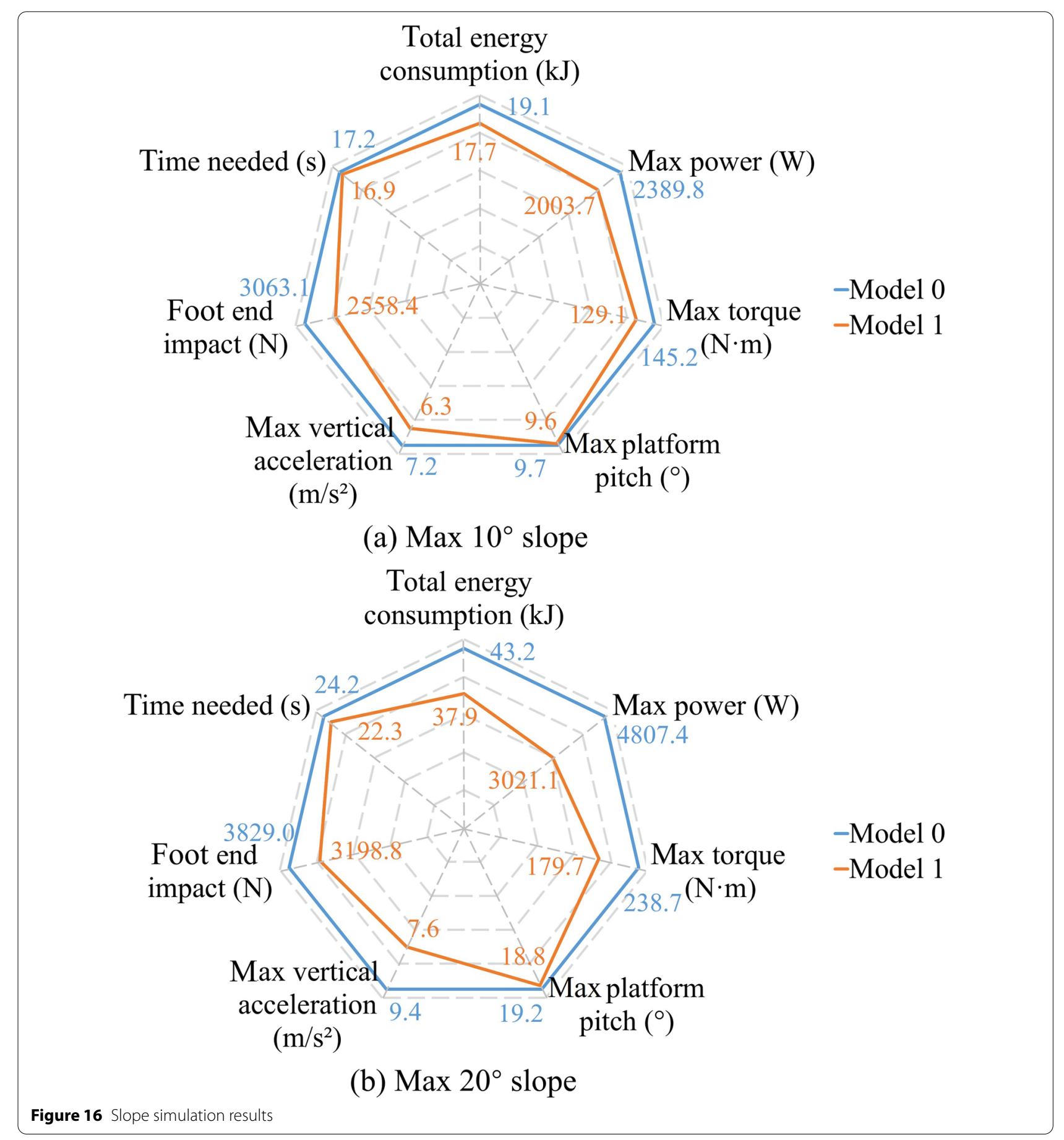

changes in velocity and acceleration. The actuation of the legs are in constant angular velocities. The properties of the material and the geometry shape of the feet have been taken into consideration in the simulation. Some other important simulation parameters are listed in
Table 5, and the simulation results of Model 1 are plotted in Figure 15.

The vertical obstacle surmounting simulation results show that Model 1 surmounted the obstacle up to 400 $\mathrm{mm}$ in height without impact and Model 0 surmounted the obstacle under $124 \mathrm{~mm}$ in height. Model 0 failed to surmount the obstacles higher than $124 \mathrm{~mm}$ regardless 


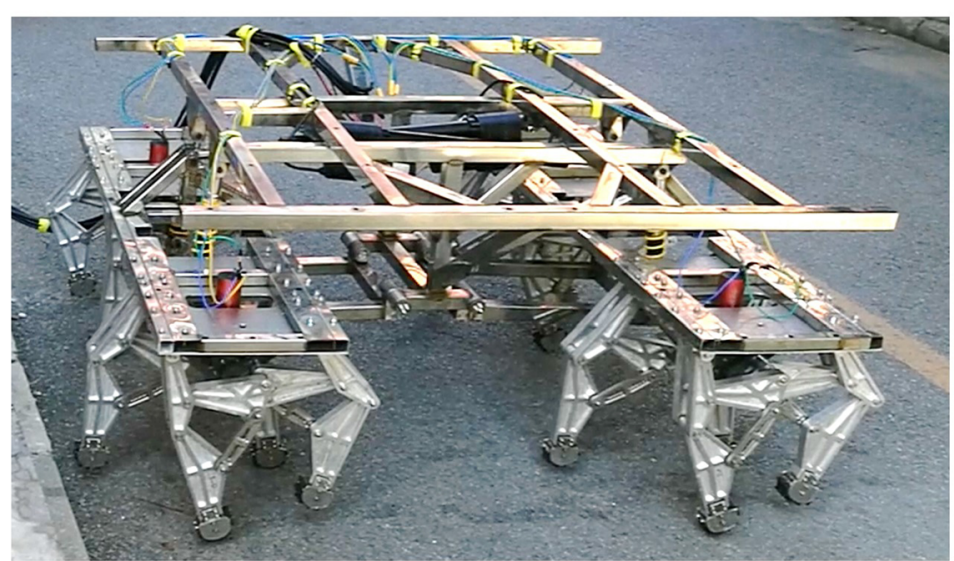

(a) Mobile platform

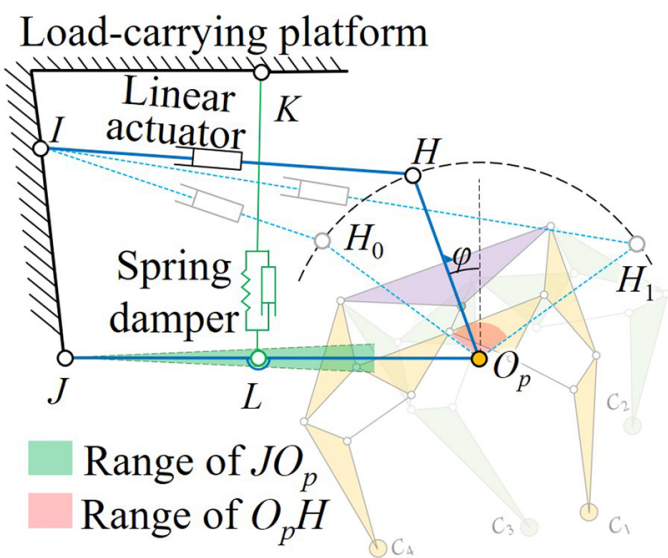

(b) Diagram of actuated joint

Figure 17 Experimental prototype

Table 6 Prototype parameters

\begin{tabular}{ll}
\hline Parameter & Value \\
\hline Dimensions $(\mathrm{mm})$ & $1500 \times 1500 \times 670$ \\
Weight $(\mathrm{kg})$ & 180 \\
Max load $(\mathrm{kg})$ & 200 \\
Major material & Aluminum alloy \\
Foot material & Rubber \\
Max speed $(\mathrm{km} / \mathrm{h})$ & 12 (on the flat ground) \\
Adjusting actuator & $450 \mathrm{~W}$ linear brushed DCM $\times 2$ \\
Leg actuator & $3 \mathrm{~kW}$ BLDCM with reducer $\times 4$ \\
Battery & $24 \mathrm{~V}, 40$ Ah, $15 \mathrm{C}$ \\
Operation mode & Remote control
\end{tabular}

of the initial state. Model 1 achieved to surmount the obstacle $400 \mathrm{~mm}$ in height with the pitch angle $\varphi_{V O}=40^{\circ}$, which agreed with the analysis.

A pair of the simulations were tested on the ground with sinusoidal slopes. For Model 1, the pitch angles adjusted to fit the ground. The results are shown in Figure 16.

The results show that on the ground with slopes, Model 1 has the better performances than Model 0 and the differences are more obvious with the larger slope angle.

\subsection{Prototype Experiments}

The experimental prototype was designed and fabricated as shown in Figure 17. Each pitch adjustment joint was actuated by a four-bar mechanism with a linear actuator. Spring dampers were introduced to absorb the impact. The parameters of the prototype are listed in Table 6.

The robot prototype is capable of the vertical obstacle up to $350 \mathrm{~mm}$ in height and the ground with $20^{\circ}$ slope, as shown in Figures 18 and 19 correspondingly.

The obstacle surmounting process was operated manually via a $2.4 \mathrm{G}$ remote control in $32 \mathrm{~s}$. According to the strategy, the obstacle surmounting process was divided into several states. Firstly, the robot stood in front on the obstacle with a proper distance, as shown in Figure 18a. Then the robot walked forwards and the front LUAP increased the pitch angle until $\varphi_{V O}=40^{\circ}$, as shown in Figure 18b. When the forefront leg stepped on the top of the obstacle, the pitch angle of the front LUAP decreased to the level state. For the pitch angle of the load carrying platform had been changed, the hind LUAP was adjusted to maintain level, as shown in Figure 18c. After that, the robot went on and the hind LUAP kept a proper distant from the obstacle, as shown in Figure 18d. Then the robot went on and the pitch angle increased to $40^{\circ}$ and the forefront foot of the hind LUAP stepped on the top of the obstacle, as shown in Figure 18e and $\mathrm{f}$. The pitch angle of the hind LUAP decreased to be level and lifted the hind LUAP up on the top of the obstacle. At the same time, the front LUAP cooperated and stayed level, as shown in Figure $18 \mathrm{~g}$. Lastly, the robot went on until the entire robot was on the obstacle, as shown in Figure 18h.

Experiment on the sloping ground was also carried out. The front and the hind LUAPs kept parallel to the ground below, and there were no idle legs. Every leg of the robot had supporting phase and the movement of the robot was smooth, as shown in Figure 19a-d. To make the comparison, a control experiment was 


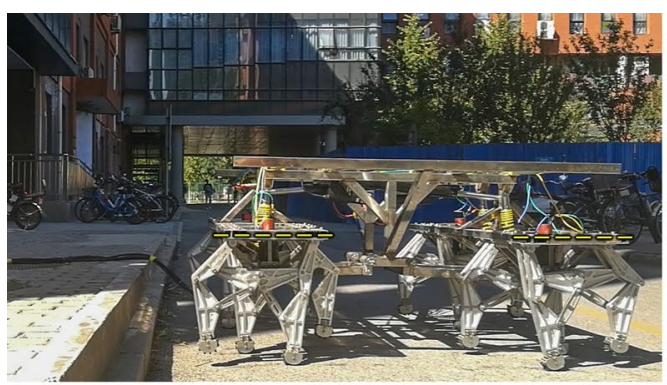

(a) $t=0 \mathrm{~s}$

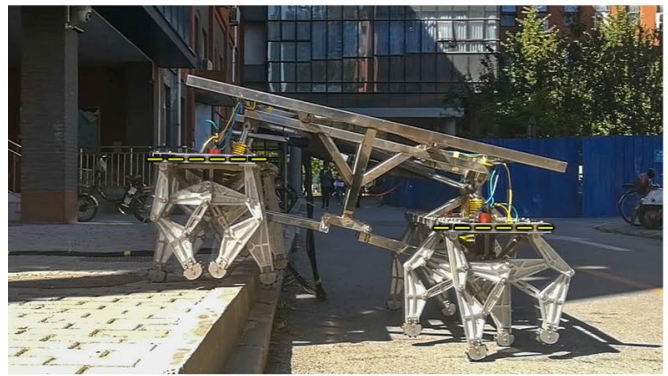

(c) $t=13 \mathrm{~s}$

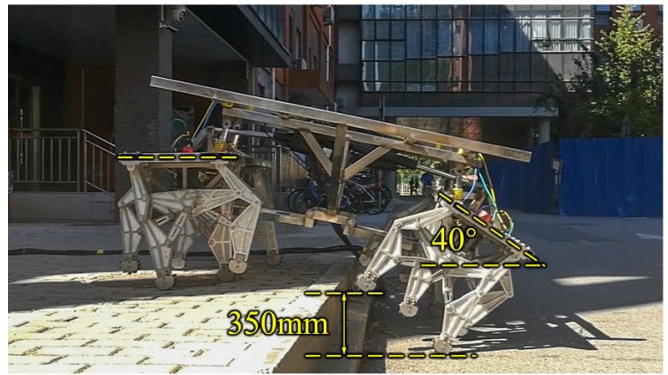

(e) $t=19 \mathrm{~s}$

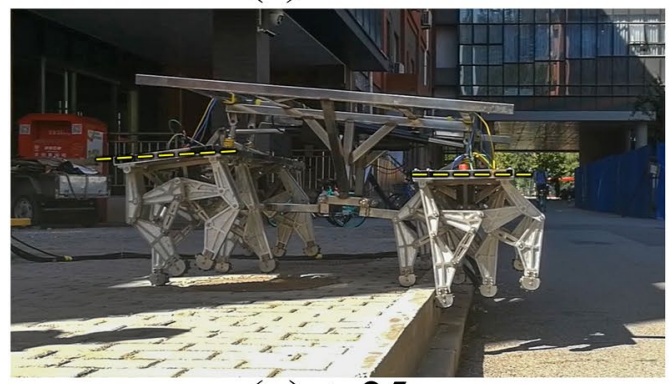

(g) $t=25 \mathrm{~s}$

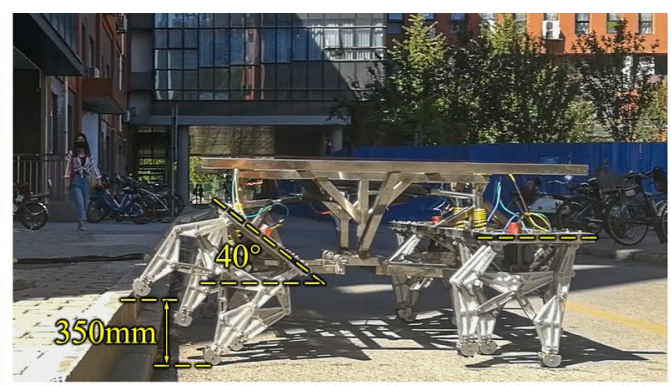

(b) $t=5 \mathrm{~s}$

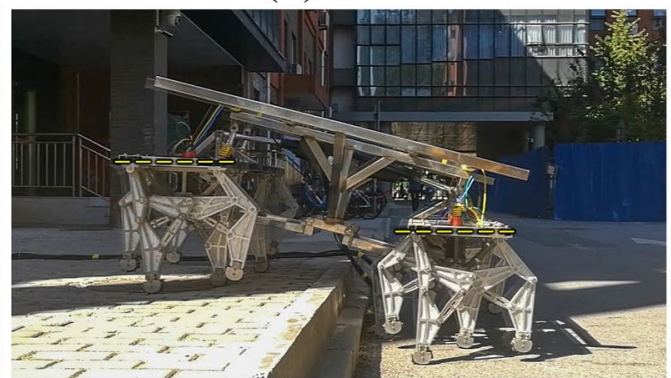

(d) $t=16 \mathrm{~s}$

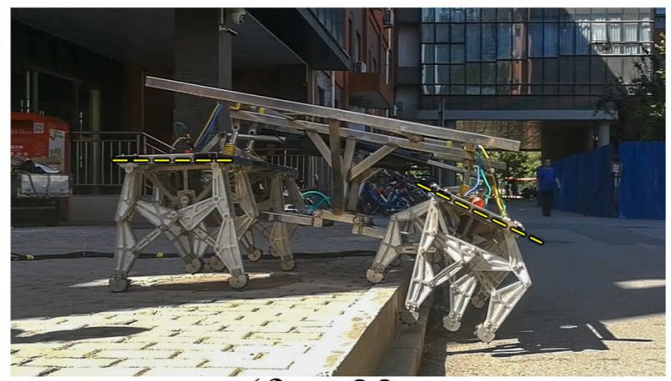

(f) $t=23 \mathrm{~s}$

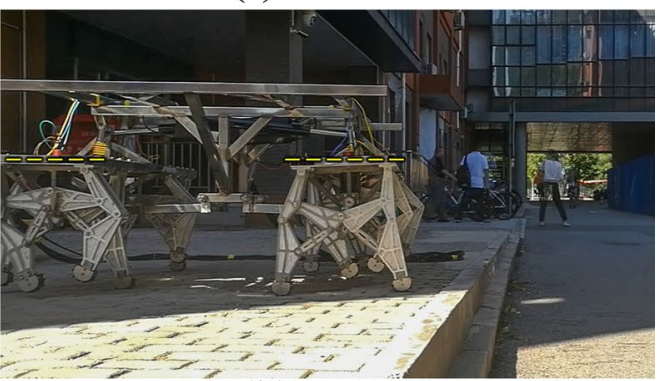

(h) $t=32 \mathrm{~s}$

Figure 18 Experiment on $350 \mathrm{~mm}$ vertical obstacle

carried out. A control experiment is also carried out and the adjustments were locked, as shown in Figure 19e-h. In the control experiment, idle legs occurred as shown in Figure 19f and g. The idle legs had no contact with the ground in a crank cycle and were not able to provide the supporting or traction forces. They also raised the duty ratio of other legs and decreased the overall mechanical efficiency. 


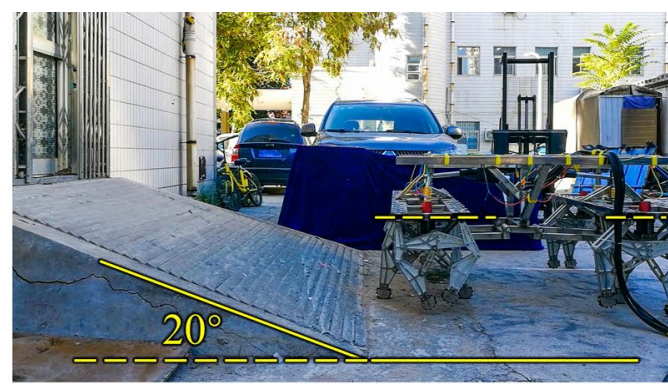

(a)

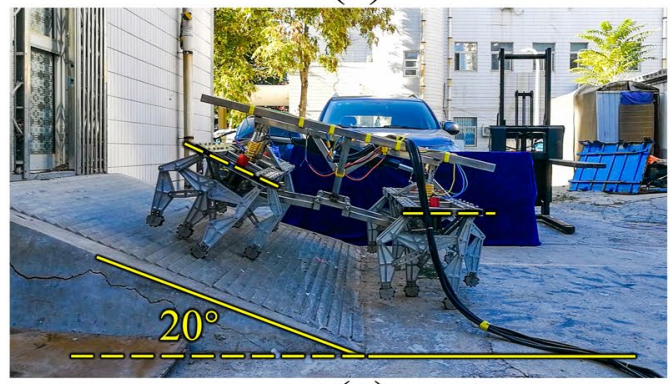

(c)

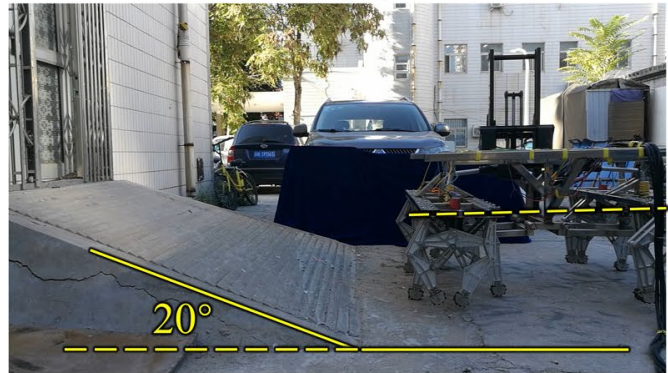

(e)

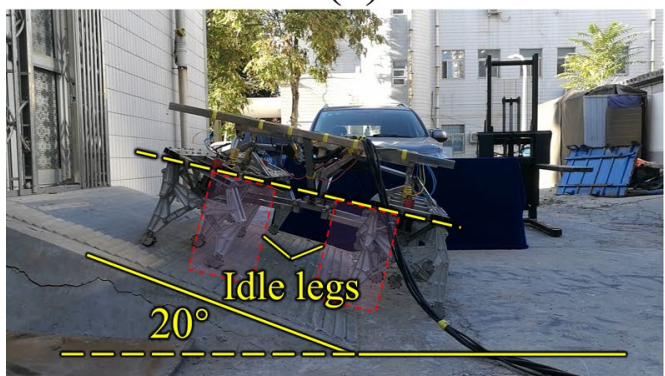

(g)

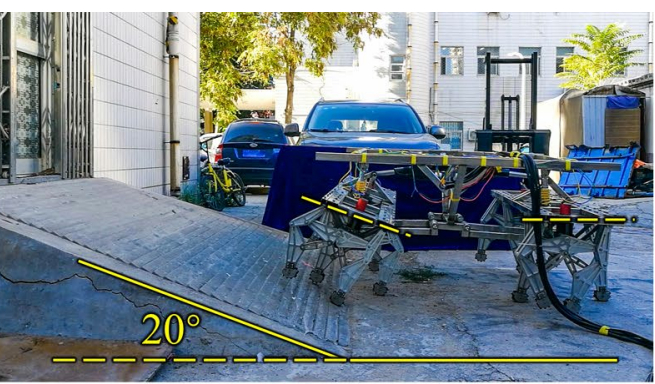

(b)

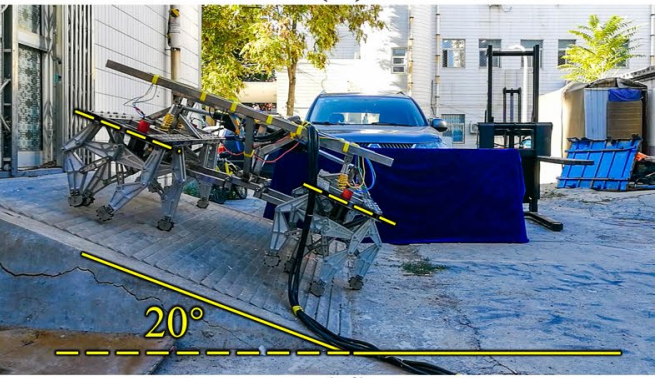

(d)

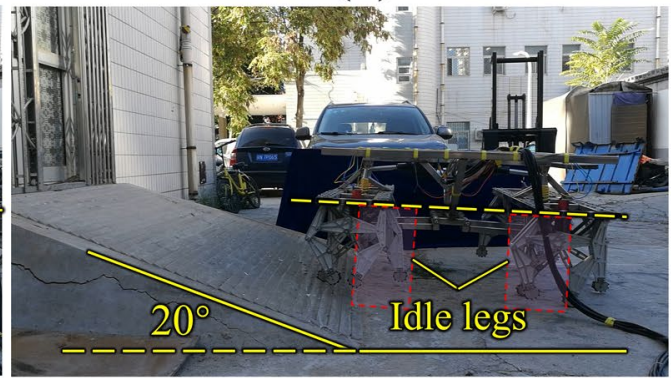

(f)

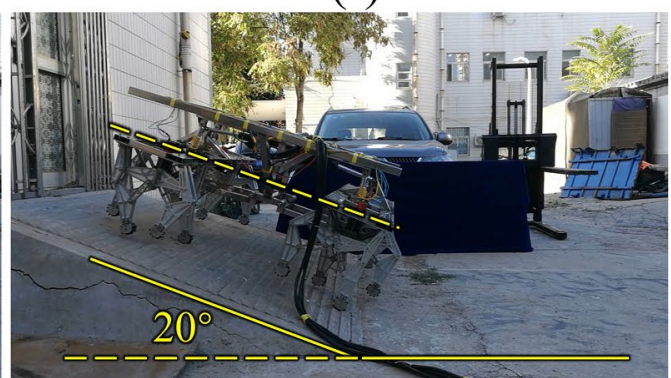

(h)

Figure 19 Experiments on $20^{\circ}$ slope

\section{Conclusions}

(1) Aiming at obstacle surmounting, a novel lowDoF multi-legged robot with pitch adjustive units was proposed.

(2) Based on the kinematic analysis and optimization of the close-chain six-bar mechanical leg, the step height, step length and the body fluctuation of B-LUAP and Q-LUAP were analysed and compared. The result showed that Q-LUAP had better performances.

(3) The vertical obstacle surmounting strategy was given. The pitch angle parameters of the platform and the LUAPs at different states were calculated. Furthermore, the recommended pitch angle was adopted based on the probability of impact. 
(4) The obstacle surmounting performance of the robot was verified and compared by carrying out the dynamic simulations and robot prototype experiments. The results showed that the robot was capable of the vertical obstacle up to $350 \mathrm{~mm}$ in height and the ground with $20^{\circ}$ slope.

\section{Acknowledgements}

The authors sincerely thanks to Professor Shuhui Zhou of Hebei Agricultural University for her critical discussion and reading during manuscript preparation

\section{Authors' Contributions}

QR was in charge of the whole trial and completed the manuscript. JW provided the core idea and the prototype design schemes. He was in charge of the prototype experiment. JW and YY modified the manuscript YY also provided the necessary equipment and sites for the experiments. All authors read and approved the final manuscript.

\section{Authors' Information}

Qiang Ruan, born in 1989, is currently a post-doctoral researcher at Beihang University, China. He received his PhD degree from Beijing Jiaotong University, China, in 2020. His research interests include mechanical design and robotics.

Jianxu Wu, born in 1989, is currently a postdoctoral researcher at Tsinghua University, China. He received his PhD degree from Beijing Jiaotong University, China, in 2019. His research interests include mechanism design, and robotics.

Yan-an Yao, born in 1972, is currently a professor and a PhD candidate supervisor at Beijing Jiaotong University, China. He received his PhD degree from Tianjin University, China, in 1999. His research interests include mechanical design and robotics.

\section{Funding}

Supported by National Natural Science Foundation of China (Grant No. 51735009).

\section{Competing Interests}

The authors declare no competing financial interests.

Received: 22 February 2020 Revised: 25 January 2021 Accepted: 22 May 2021

Published online: 15 June 2021

\section{References}

[1] S Nansai, N Rojas, M R Elara, et al. A novel approach to gait synchronization and transition for reconfigurable walking platforms. Digital Communications and Networks, 2015, 1(2): 141-151. https://doi.org/10. 1016/j.dcan.2015.04.003.

[2] M H Raibert. Legged robots. Communications of the ACM, 1986, 29(6): 499-514. https://doi.org/10.1145/5948.5950.

[3] B Wang, C Zhou, Z Duan, et al. Untethered quadrupedal hopping and bounding on a trampoline. Frontiers of Mechanical Engineering, 2019, 15(2): 1-12. https://doi.org/10.1007/s11465-019-0559-5.

[4] H C Zhuang, H B Gao, Z Q Deng, et al. A review of heavy-duty legged robots. Science China Technological Sciences, 2014, 57(2): 298-314. https://doi.org/10.1007/s11431-013-5443-7.

[5] Y Pan, F Gao. Payload capability analysis of a new kind of parallel leg hexapod walking robot. Proceedings of the 2013 International Conference on Advanced Mechatronic Systems, IEEE, 2013: 541-544. DOI: https://doi.org/10.1109/ICAMECHS.2013.6681703.
[6] X B Chen, F Gao, C K Qi, et al. Spring parameters design for the new hydraulic actuated quadruped robot. Journal of Mechanisms and Robotics, 2014, 6(2). https://doi.org/10.1115/1.402575.

[7] M H Raibert. Dynamic legged robots for rough terrain. Proceedings of 10th IEEE-RAS International Conference on Humanoid Robots, IEEE, 2010: 1-1. DOI: https://doi.org/10.1109/ICHR.2010.5686280.

[8] M J A Tenreiro, M Silva. An overview of legged robots. Proceedings of International Symposium on Mathematical Methods in Engineering, 2006: 1-40.

[9] M H Raibert, K Blankespoor, G Nelson, et al. Bigdog, the rough-terrain quadruped robot. IFAC Proceedings Volumes, 2008, 41(2): 10822-10825.

[10] R Playter, M Buehler, M H Raibert. BigDog. In: Society of Photo-Optical Instrumentation Engineers (SPIE) Conference Series, 2006. DOl: https:// doi.org/10.1117/12.684087.

[11] M H Raibert. Alphadog, the Rough-Terrain Robot. Adaptive Mobile Robotics, 2012: 7-7. DOI: https://doi.org/10.1142/9789814415958_ 0005.

[12] P Fankhauser, M Hutter. ANYmal: a unique quadruped robot conquering harsh environments. Research Features, 2018(126): 54-57.

[13] J Hwangbo, J Lee, A Dosovitskiy, et al. Learning agile and dynamic motor skills for legged robots. Science Robotics, 2019, 4(26): eaau5872.

[14] G Bledt, M J Powell, B Katz, et al. MIT cheetah 3: Design and control of a robust, dynamic quadruped robot. Proceedings of IEEE/RSJ International Conference on Intelligent Robots and Systems (IROS), 2018 : 2245-2252. https://doi.org/10.1109/IROS.2018.8593885.

[15] L Fang, F Gao. Type design and behavior control for six legged robots. Chinese Journal of Mechanical Engineering, 2018, 31:59. https://doi.org/ 10.1186/s10033-018-0259-9.

[16] Y Sugahara, T Hosobata, Y Mikuriya, et al. Realization of dynamic human-carrying walking by a biped locomotor. Proceedings of IEEE International Conference on Robotics and Automation, 2004, 3: 3055-3060.

[17] L Birglen, C Ruella. Analysis and optimization of one-degree of freedom robotic legs. Journal of Mechanisms and Robotics, 2014, 6(4).

[18] K Komoda, H Wagatsuma. Energy-efficacy comparisons and multibody dynamics analyses of legged robots with different closed-loop mechanisms. Multibody System Dynamics, 2017, 40(2): 123-153.

[19] D Fedorov, L Birglen. Analysis and design of a two degree of freedom Hoeckens-Pantograph leg mechanism. ASME 2015 International Design Engineering Technical Conferences and Computers and Information in Engineering Conference, 2015, 5B(39). https://doi.org/10.1115/DETC2 015-47330.

[20] T Jansen. The great pretender. Rotterdam: 010 Publishers, 2007.

[21] C Liang, M Ceccarelli, Y Takeda. Operation analysis of a ChebyshevPantograph leg mechanism for a single DOF biped robot. Frontiers of Mechanical Engineering, 2012, 7(4): 357-370. https://doi.org/10.1007/ s11465-012-0340-5.

[22] N G Lokhande, V B Emche. Mechanical spider by using Klann mechanism. International Journal of Mechanical Engineering and Computer Applications, 2013, 1(5): 13-16.

[23] Y Pan, F Gao. Position model computational complexity of walking robot with different parallel leg mechanism topology patterns. Mechanism and Machine Theory, 2017, 107: 324-337. https://doi.org/ 10.1016/j.mechmachtheory.2016.09.016.

[24] H S Park, S Floyd, M Sitti. Roll and pitch motion analysis of a biologically inspired quadruped water runner robot. International Journal of Robotics Research, 2010, 29(10): 1281-1297. https://doi.org/10.1177/ 0278364909354391.

[25] J Park, J Lee, J Lee, et al. Raptor: Fast bipedal running and active tail stabilization. Proceedings of 11 th International Conference on Ubiquitous Robots and Ambient Intelligence (URAI), IEEE, 2014: 215-215. https://doi. org/10.1109/URAI.2014.7057424.

[26] J X Wu, Y A Yao, Y Li, et al. Design and analysis of a sixteen-legged vehicle with reconfigurable close-chain leg mechanisms. Journal of Mechanisms and Robotics, 2019, 11(5): https://doi.org/10.1115/1.40440 03.

[27] J X Wu, Y A Yao. Design and analysis of a novel walking vehicle based on leg mechanism with variable topologies. Mechanism and Machine Theory, 2018, 128: 663-681. 
[28] D B Byers. Multifunctional Utility/Logistics and Equipment (MULE) Vehicle Will Improve Soldier Mobility, Survivability and Lethality. Acquisition Logistics and Technology, Fort Belvoir Va, 2008.
[29] B M Yamauchi. PackBot: a versatile platform for military robotics. International Society for Optics and Photonics, 2004, 5422: 228-237. https:// doi.org/10.1117/12.538328.
Submit your manuscript to a SpringerOpen ${ }^{\odot}$ journal and benefit from:

- Convenient online submission

- Rigorous peer review

- Open access: articles freely available online

- High visibility within the field

- Retaining the copyright to your article

Submit your next manuscript at $\boldsymbol{\nabla}$ springeropen.com 